\title{
Sexo sin palabras. La función del silencio en el intercambio sexual anónimo entre hombres ${ }^{1}$
}

\section{Sex without words. The role of silence in anonymous sex encounters between men}

\author{
Jose Antonio LANGARITA ADIEGO \\ Universidad de Girona. Universidad de Barcelona \\ josan.langarita@udg.edu
}

Recibido: 13 de junio de 2012

Aceptado: 27 de marzo de 2013

\begin{abstract}
Resumen
El intercambio sexual anónimo entre hombres es una práctica habitual en diferentes espacios públicos. Esta actividad se desarrolla en una coyuntura marcada por una segregación sexual en la que la lógica de la heterosexualidad se convierte en hegemónica y hace que aquellas conductas o deseos que se escapen del binomio hombre/mujer sean desacreditados y objeto de sanción. El intercambio sexual anónimo se presenta como una práctica que responde a la necesidad de satisfacer los deseos entre hombres en un contexto de hostilidad sexual. Este intercambio, como cualquier otra actividad social, esta marcado por unas normas y consensos que regulan la relación, dejando al silencio un lugar primordial para poder garantizar el máximo anonimato posible entre los participantes. De este modo, el silencio se convierte en una herramienta indispensable para el acuerdo sexual y para la garantía del secreto, trasladando así el discurso dominante que invisibiliza la diferencia sexual al proceso de negociación y acuerdo entre los hombres que participan en este tipo de interacción.
\end{abstract}

Palabras clave: homosexualidad, homofobia, ritual de interacción, silencio, anonimato.

\begin{abstract}
Anonymous sexual exchange between men is a common practice in various public spaces. The practice flourishes in a context defined by sexual segregation - a context in which heterosexual logic becomes hegemonic and discredits and sanctions all behaviors and desires that lay outside the man/woman binomial. As a practice it is a response that fulfills the necessity for sexual desire between men in a sexual context which is hostile towards them. This exchange, like any other social activity, is regulated by norms and consensus, of which silence is one of the most fundamental as it guarantees the maximum anonymity between participants. Thus silence becomes an indispensable tool within the sexual contract as a guarantee of secrecy while at the same time translating the dominant discourse which makes sexual difference invisible into a process of negotiation and agreement between men who participate in this kind of interaction.
\end{abstract}

${ }^{1}$ Debo agradecer las aportaciones a este trabajo realizadas por Joan Bestard, Manuel Delgado, Oscar Guasch y Laura Villaplana. Así mismo han sido también vitales las consideraciones de los miembros de la Línea de investigación en torno a los cuerpos, géneros y sexualidades del GRECS. 
Keywords: homosexuality, homophobia, ritual interaction, silence, anonymity.

Referencia normalizada: Langarita Adiego, JA. (2013) Sexo sin palabras. La función del silencio en el intercambio sexual anónimo entre hombres, en Revista de Antropología Social, 22: XX-XX

SUMARIO: 1. Introducción. 2. Metodología. 3. Del silencio de la homosexualidad. 4. Del silencio del homosexual. 5. Del silencio como estrategia comunicativa para el sexo anónimo. 6. Conclusiones. 7. Referencias bibliográficas.

\section{Introducción}

Las zonas de encuentro sexual entre hombres en espacios urbanos son conocidas desde al menos el siglo XVII (Hahn, 1973; Silabis, 1999). Por su parte Francisco Vazquer Garcia y Richard Cleminson afirman que la "existencia de enclaves para los encuentros sexuales entre varones era algo intrínseco a la vida homosexual en los núcleos urbanos europeos a partir de la baja edad media y especialmente en el periodo moderno" (2010[1997]: 268). Estos precedentes dan cuenta de una forma de relación entre hombres dirigida hacia el encuentro sexual en espacios públicos que todavía permanece en uso. La práctica de sexo anónimo conocida como cruising ha sido definida por Michael Reece y Brian Dodge (2004) como "el ritual de búsqueda e interacción con potencial de pareja sexual que suelen ser desconocidos para los participantes". Por lo tanto la práctica del cruising no refiere únicamente al acto sexual, sino también al artefacto social que conduce hacia él.

Los estudios del sexo anónimo tomaron presencia fundamentalmente a partir del trabajo de Laud Humphreys (1975 [1970]), y posteriormente de Meredith R. Ponte (1974), y Richard R. Troiden (1974), quienes mantenían un discurso de criminalidad y desviación de los sujetos que participaban en este tipo de actividad. Sin embargo, la llegada del VIH transformó la mirada de las zonas de intercambio sexual anónimo y promovió un interés basado en el estudio para la prevención de la enfermedad y el reconocimiento de las comunidades LGTB. En este sentido tomaron partido trabajos como los de Oscar Guasch (1991), Paul Flowers, Graham Hart y Claire Marriott (1999), Jonathan D. Huber y Peggy J. Kleinplatz (2002), William Elwood, Kathryn Greene y Karen Carter (2003), Richard Tewksbury (2008), Fernando Villaamil y Maria Isabel Jociles (2008; 2011) y Fernando Lores (2012) entre otros. Estas investigaciones han ido abriendo un espacio de análisis desde los años noventa y han iniciado un nuevo camino marcado por el respeto a los sujetos que participan en este tipo de actividades. Lo que ha permitido un acercamiento al estudio de la sexualidad desde otros escenarios.

El trabajo que viene a continuación se presenta como una contribución a estos estudios sobre el sexo anónimo desde un análisis de la comunicación en la práctica del cruising en espacios públicos no comerciales. De manera que se pretende estudiar los diferentes significados que adquiere la figura del silencio en relación a la homosexualidad y los usos que se le atribuye en estas zonas de ligue para hombres fuera del circuito comercial. 
En este trabajo se presenta el silencio en tres formas diferentes que se interrelacionan para articular y mantener las lógicas sexuales hegemónicas, que inequívocamente está marcada por el paradigma de la heterosexualidad.

Por un lado se muestra el silencio como una herramienta que permite invisibilizar una realidad ante el conjunto de la sociedad. Es decir, como una maniobra de evitación, como un ejercicio que permite situar la desviación en la alteridad. En segundo lugar, se aborda el silencio como una estrategia que permite ocultar aquello que no es aceptado socialmente. Como una táctica de supervivencia de los hombres que mantienen sexo con otros hombres. En este caso con la finalidad de evitar el estigma o la sanción por aquello que son. Y finalmente el silencio como una estrategia, una forma de anonimato, como un elemento que facilita las relaciones sexuales entre los hombres que, en principio, no se conocen.

\section{Metodología}

Este trabajo de investigación se llevó a cabo principalmente en el parque de Montjuïc de Barcelona entre los años 2009 y 2012. No obstante, se realizaron inmersiones de campo en otros escenarios de intercambio sexual anónimo como la playa de la Mar Bella, en Barcelona, la zona del bosque y la de la playa de Sitges, a cuarenta y un kilómetros de Barcelona, y en la pineda de Gavà en área metropolitana de la ciudad. Las zonas comerciales para la interacción sexual entre hombres como saunas o cuartos oscuros han sido objeto de análisis de investigadores como Weinberg y Williams (1975), Allan Bébubé (1996), Ross Higgins (1999), Ira Tattelman (1999), Fernando Villaamil y Maria Isabel Jociles $(2008,2011)$ o Camilo Albuquerque de Braz (2003). Sin embargo en este trabajo no han sido incorporados este tipo de espacios orientados al encuentro sexual entre hombres dentro de locales con finalidades comerciales y se ha centrado en conocer las estrategias de comunicación entre hombres que conducen al acto sexual en espacios públicos de acceso gratuito, como son parques y playas. Así mismo, tampoco se ha profundizado en las relaciones que se establecen en urinarios, ya que en el parque, playas y aparcamientos se dispone de más tiempo para la deliberación y negociación que precede al acto sexual (Tewksbury, 2008). Tal y como plantea Fernando Lores (2012), tanto los lugares de encuentros sexuales comerciales como aquellos que se llevan a cabo en espacios públicos se han dualizado y se trata de escenas sexuales diferenciadas con particularidades propias. .

Para desarrollar el trabajo de investigación he utilizado las dos técnicas fundamentales de la etnografía: la observación participante y la entrevista. La primera de ellas ha sido elemental para profundizar en una buena parte de los pormenores de las relaciones del sexo anónimo. La observación participante me ha permitido dibujar los recorridos, comprender las estrategias y establecer los elementos de interpretación que puedan explicar, al menos en parte, los procesos comunicativos que se dan en las zonas de cruising. Es por ello, por lo que he introducido el sexo como un recurso para la investigación etnográfica, lo que me ha llevado a formar parte de los acuerdos sexuales con otros participantes, así como a incorporar las normas comunicativas en la interacción. En las zonas de cruising, la observación participante permite empaparse del talante sexual que acompaña el proceso de intercambio. Esta 
aplicación, por lo tanto, es producto de una reflexión y un planteamiento teórico de partida, en el que el sexo es pensado como un ejercicio cultural que responde a las normas y estructuras de un modo de organización social. Es por ello por lo que participar de él, se ha convertido en un elemento básico para conocerlo y describirlo. Mantenerse al margen de prácticas sexuales, puede dejar de lado al investigador en una parte importante de la vida social de su objeto de estudio. Este uso podría llevar al malentendido y producir una reacción vituperadora entre los conocedores de este trabajo. Sin embargo, debo advertir, que si aceptamos que el sexo es un hecho cultural, deberíamos de poder incorporarlo a nuestra práctica etnográfica como un elemento más del análisis. Y por lo tanto esta decisión metodológica no debería ser más cuestionada cuando se trata de prácticas sexuales libres y acordadas que si se tratase de cualquier otro ámbito cultural. En este sentido, he realizado veinticuatro visitas registradas en un diario de campo con una media de dos horas de estancia: catorce al parque de Montjuïc, cinco a Sitges, tres a la pineda de Gavà, una a la playa de la Mar Bella, y una a los baños de una estación de transporte público.

Por otro lado, la entrevista ha sido una técnica mucha más difícil de utilizar en el trabajo de campo debido a la dimensión que toma el lenguaje verbal en las zonas de cruising. He llevado a cabo un total de trece entrevistas: a ocho participantes de las zonas de cruising, tres a asociaciones de derechos LGTB, a una entidad de prevención de enfermedades de transmisión sexual con un programa de intervención en la zona de cruising y una a la policía autonómica. Debo reconocer que la selección de los participantes entrevistados ha respondido más a su voluntad de acceder a la entrevista, que a un proceso selectivo del tipo de personas a las que entrevistar. Este hecho me ha llevado a entrevistar fundamentalmente a personas usuarias de las zonas de cruising que se reconocen homosexuales y que no tienen inconveniente en hacer visible su orientación sexual, aunque no acostumbran a hacer pública su participación de la actividad del cruising.

Una buena parte de la información se ha obtenido mediante conversaciones informales que he mantenido a la entrada o salida de las zonas de intercambio sexual. Las cuales han quedado registradas en las anotaciones del diario de campo. Estas conversaciones, aunque en muchas ocasiones breves, han sido un elemento fundamental que ha contribuido a orientar la mirada y a profundizar en los discursos y percepciones de los participantes.

\section{Del silencio de la homosexualidad}

No cabe duda de que el silencio es un hecho que ha acompañado a la homosexualidad en un intento constante de afirmar su inexistencia o como una estrategia para la negación de esta realidad. Desde algunas perspectivas, se podría considerar que esta afirmación corresponde a una diatriba del pasado o incluso que se trata de un lenguaje trasnochado que ya debe quedar en desuso. Sin embargo existen múltiples situaciones y experiencias que trasladan al presente lo inapropiada que resulta todavía hoy la homosexualidad para el discurso sexual hegemónico.

En este artículo no se pretende hacer una historia social de la homosexualidad, ya que existen un buen número de antropólogos, sociólogos e historiadores que han 
hecho un trabajo excepcional respecto a esta cuestión (ver Boswell, 1992 [1980]; Coll-Planas, 2010; Eribon, 2001[1999] y 2004; Foucault, 2005[1976]; Guasch, 1991; Mira, 2007 [2004]; Rubin, 1989 [1984]; Vaquez Garcia y Cleminson, 2010 [1997]; Vazquez Garcia y Moreno, 1997; Weeks, 1993). Pero sin embargo sí que es oportuno recordar que la visibilidad afectiva entre hombres continua siendo motivo de escándalo público tal y como refleja el diagnóstico de las realidades de la población LGTB en la ciudad de Barcelona (Coll-Planas y Misse, 2009) o los informes de organismos internacionales por la garantía de derechos humanos que dan cuenta de la persecución a la población homosexual alrededor de todo el mundo.

Es importante tener en cuenta que la educación familiar se ejerce mayoritariamente desde una perspectiva en la que se obvia, o se intenta contener la posible homosexualidad de nuestros pequeños con la finalidad de evitar la desviación incorregible o la visibilidad de una sexualidad no legitimada (ver Herdt y Koff, 2002 [2000]; Weston, 2003 [1997], Pichardo Galan, 2009a, 2009b). En el prólogo a la edición española del libro Gestión familiar de la homosexualidad de Hedt y Koff, el antropólogo catalán Oscar Guasch (2002) asegura que la homosexualidad es algo que los hijos confiesan a sus padres, no que se explica, ya que constituye algo indeseable y resulta la prueba de un fracaso en el proyecto familiar, de un sueño inalcanzado. Evidentemente, no cabe duda de que algunas familias han introducido en su repertorio educativo discursos relativos a la diversidad sexual. Pero sin embargo, en ocasiones, acostumbran a mantenerse los discursos de ocultamiento e invisibilidad tanto en el entorno escolar como familiar (Platero Méndez, 2008). La familia es inequívocamente el primer espacio donde el silencio se articula, se mantiene y se reafirma para contribuir a la causa heterosexual mediante la invisibilización de los deseos entre personas del mismo sexo. De manera que la homosexualidad en la familia es vivida como un hecho vergonzante no solo en la esfera pública sino que también en el ámbito doméstico donde suele causar un gran impacto en el momento en el que se descubre.

Por otro lado, aunque sin intención de entrar en pormenores, el entorno laboral, los vecinos o el pueblo en general, cuando se trata de zonas rurales, son otros espacios donde la norma del silencio queda manifiesta y consensuada por el conjunto social. Es por ello por lo que podemos afirmar que existen múltiples situaciones que nos llevan a constatar este pacto de silencio en nuestro día a día y especialmente presente para muchos homosexuales que constantemente deben realizar ejercicios para ocultar aquello que son con la finalidad de garantizar su permanencia en la esfera social en un marco de respeto y de consideración.

Sin embargo, de ningún modo podemos pensar que la norma del silencio, o mejor dicho la obligatoriedad de silenciar, se genera de manera autónoma, sin pretexto ni condiciones, como si de un hecho dado se tratase. En realidad, tal y como anuncia Foucault (2005 [1976]), podemos entender que es el discurso el que produce y obliga al silencio. Se trata de la paradoja de decir aquello de lo que a su vez se prohíbe hablar. Este discurso puede ser lejano o desconocido para muchos actores que se someten a la norma, que aceptan aquello que se dijo una vez y ya no puede volverse a nombrar, ni tan siquiera pensar, como si se tratase de algo absoluto 
que no deja lugar para el debate ni la sospecha. El discurso explícito ha sido una herramienta que ha permitido mantener el silencio en la esfera social respecto a las sexualidades no-heterosexuales. Y aunque son diversos los actores que han contribuido en esta empresa, cabe destacar el papel del cristianismo y posteriormente el de la biomedicina, que amparadas en un valor divino o científico han hecho una gentil contribución al discurso del silencio y al mantenimiento legítimo de las estructuras heterosexuales. Sin embargo, la instauración de la ley del silencio no es el resultado de un hecho único y aislado del pasado que permanece en el presente. La norma requiere de constantes ejercicios que reafirmen su vigencia, estructuras sociales que constantemente recuerden la existencia e importancia de esta norma adquirida. Es por ello por lo que las doctrinas sexuales hegemónicas han requerido de diferentes estrategias para avalar su persistencia. La injuria, la mofa, la tortura, la cárcel, o incluso la pena de muerte han sido herramientas auxiliares que recuerdan y contribuyen a que cada persona administre en su cuerpo la dosis correspondiente de sumisión al consenso sexual. Todos los cuerpos han aprendido que la homosexualidad no es un hecho deseable, los chistes de maricones, el desprecio a lo afeminado, los insultos al diferente sexual, o el maltrato a los desviados han instaurado el silencio en cada uno de los cuerpos que comparten el espacio social.

\section{Del silencio del homosexual}

El deber de este silencio obliga a las personas a tomar caminos vitales que sirvan de coartada para evitar el descubrimiento fatídico. Abonarse al binomio hombre/ mujer, al patrón de reproducción y favorecer la injuria del diferente, suelen ser buenas estrategias para evitar la duda o el examen de cumplimiento del contrato social (Wittig, 2006 [1992]).

El silencio es un fenómeno que ha acompañado la vida de gays y lesbianas de manera constante y repetida. Cualquier homosexual se ha visto obligado a ocultarse, pasar por heterosexual en diferentes contextos o fases de su vida. Conocedores de que aquello que son no está bien, los homosexuales han ocultado todo aquello que merece ser guardado en secreto. En la misma línea que señala Didier Eribon (2001[1999]), considero que salir del armario no es una acción única y definitiva, cualquier homosexual volverá a él cuando necesite presentarse ante una entrevista de trabajo, cuando se sitúe ante un auditorio en el que debe dar un aspecto de seriedad y responsabilidad, o en cualquier otra situación que lo requiera. El armario es un peso que cualquier gay lleva consigo para poder volver a meterse de inmediato si el contexto lo exige. Es por ello por lo que el secreto y el silencio son los cómplices constantes de cualquier homosexual. Permanecer en silencio, es una estrategia práctica y vital para muchas personas que les permite mantener una vida social, en principio, sin incidentes ni altercados. El armario, pensado de este modo, forma parte integral del discurso del silencio, envolviendo al sujeto en un arquetipo diferencial que no ha sido elegido pero que debe ocultarse para favorecer un desarrollo correcto de la vida en sociedad.

El hecho de permanecer en el armario es en sí mismo un comportamiento que se ha iniciado como tal por el acto discursivo del silencio, no un silencio concreto, sino 
un silencio que va adquiriendo su particularidad, a trancas y barrancas, en relación con el discurso que lo envuelve y lo constituye de forma diferencial. (Sedgwick, 1998 [1990]: 14)

Hacer pública la condición de homosexualidad, de algún modo, implica asumir todas las características que se han asociado al otro homosexual, y es por ello por lo que muchos gays se encuentran sujetos a una identidad prefabricada, que acostumbra a generar categorías estancas que poco tienen que ver con las experiencias vividas. Esta identidad publica se ha construido a partir de una visibilidad basada en la fiesta, el consumo y el ocio, que aunque es recurrente para algunas personas, no hace justicia al ocultamiento y silencio que forma parte en un momento u otro de la vida de la mayor parte de los homosexuales.

Bien es cierto que en las ultimas décadas, la población homosexual ha constituido un grupo de presión en tanto que se han organizado en asociaciones de defensa de derechos civiles y también por un creciente poder adquisitivo (Bailey, 1999). Un aumento en el consumo por parte de la población gay ha permitido la legitimación de otros estilos de vida sexual que han pasado de viciosos a consumidores. Nuevas instituciones dirigidas al publico gay copan algunos barrios de las grandes ciudades construyendo así otra hegemonía sexual basada en una cultura homonormativa (Duggan, 2002). Es decir generando patrones de comportamiento, deseo, ocio y estilo de vida que debe ser compartido por todos aquellos sujetos a los que se les atribuye la categoría homosexual. Esta institucionalización del deseo sexual ha generado una dinámica propia de inclusión y exclusión, de manera que aquellos sujetos que no reúnen los atributos económicos o sociales para poder sumarse al circuito gay legitimado, ven como sus expresiones sexuales deben mantenerse en el ocultamiento o trasladarse a lo que Fernado Lores (2012) ha llamado mercados periféricos.

No obstante, ninguna norma social esta dotada de una rigidez tan completa que impida quebrantarla, destruirla o suplantarla. Siempre quedan fisuras sin resolver, nudos sin atar o acuerdos sin cerrar que dejan un espacio para la fuga. La norma de ningún modo puede ser absoluta y es por ello por lo que la ley del silencio no evita la experiencia homosexual, aunque evidentemente contribuye a su estigmatización, sanción y exclusión. A pesar del hecho vergonzante, de los discursos apocalípticos y de las sanciones sociales, la homosexualidad continua siendo una práctica cotidiana de muchas personas para el gozo, el placer y la satisfacción sexual. Ahora bien, debemos puntualizar que la instauración del silencio ha sido un elemento clave para la configuración de la escena homosexual, y a la vez ha permitido la convivencia simultánea del gozo y el anonimato entre los asistentes a diferentes locales, saunas o zonas de intercambio sexual anónimo. En este último caso, el silencio toma una dimensión más práctica que sancionadora, e intenta cumplir como objetivo fundamental el anonimato de los asistentes, aunque también tiene otras funciones asociadas.

\section{Del silencio como estrategia comunicativa para el sexo anónimo}

Cualquier persona que asista por primera vez a una zona de cruising, lo que inicialmente le llamará la atención será probablemente el modo en que se organiza la 
comunicación entre los participantes sin el uso de la palabra para el acuerdo y la práctica sexual. Los usuarios pueden llevar a cabo sin ningún tipo de problemas el ritual de intercambio sexual anónimo en silencio, sin establecer una conversación verbal con el resto de sus parejas sexuales, e incluso cuando el acto sexual se ha consumado.

Humphreys (1970), uno de los primeros teóricos en interesarse por el estudio del intercambio sexual anónimo, apunta que excepto para la masturbación el sexo necesita de la acción colectiva; es decir, necesita de la colaboración de dos personas como mínimo. A su vez, la acción colectiva requiere inevitablemente de la comunicación para poder llevarse a cabo. Es por ello por lo que en las zonas de cruising se ha establecido un modo de comunicación particular que permite desarrollar la acción colectiva en silencio con la finalidad, en este caso, de obtener los beneficios del placer sexual. El anonimato suele ser una constante entre los intereses de los usuarios y puede generar una preocupación extrema para algunos de ellos que en ocasiones se tapan el rostro a lo largo del ritual de interacción sexual con pañuelos, gorra o incluso cascos de moto para no poder ser reconocidos. "Uno no conoce a quien toca, azota o se la mama, pero siente adoración por él” (Tattelman, 1999: 88-89).

El silencio, en las zonas de cruising, es una herramienta que las personas utilizan para intentar presentarse solamente en algunos aspectos de sí mismos, así el individuo trata de hacer visible únicamente aquellos elementos que le favorecen para el intercambio sexual. De alguna manera, como apuntan Elwood, Greene, y Carter (2003), así como Delph (1978), el silencio ayuda a los participantes a poder separar lo que pertenece a su vida social de la práctica del sexo anónimo. La ausencia de palabras es una buena estrategia para minimizar los efectos negativos de los significados estigmatizantes de la homosexualidad. Es por ello por lo que el cruising requiere de una interacción que emita las menores revelaciones posibles respecto a los sujetos que están participando en ella, de manera que en silencio las explicaciones o decisiones no merecen matizaciones. En este contexto comunicativo, los usuarios hacen públicos al resto de participantes aquellos aspectos más corporales y cargados de significación sexual, pero intentan restringir las informaciones personales relativas al nombre, origen, edad, lugar de residencia, y otros datos que suelen intercambiarse en el coqueteo que se da en otros escenarios sociales.

El silencio cumple la función de ocultamiento en un sentido amplio. Por un lado, de manera íntima y personal, evitando ofrecer informaciones adicionales ajenas a la interacción sexual y que únicamente entorpecerían el acuerdo. Y por otro, también puede evitar el descubrimiento o sospecha de la actividad que se está llevando a cabo por parte de aquellos viandantes con los que se comparte espacio con finalidades diferentes. Una interacción en la que tan apenas se producen ruidos, es mucho más difícil de ser advertida por aquellas personas que están presentes en el lugar donde se producen, así muchas de las personas que pasean por los parques, playas, o entran a los lavabos públicos con otra finalidad no sexual, no suelen reconocer el ritual de interacción que se está llevando a cabo por quienes le rodean.

Cuando en el proceso de negociación, así como en el propio acto sexual no existen las palabras, la información recibida por los participantes se establece 
habitualmente a partir de los canales visuales que instigan a la interacción (Douglas y Tewksbury, 2008). De manera que el individuo tiene más dificultad para recordar con quién ha tenido sexo cuando el intercambio de palabras no se ha producido.

Debemos entender el silencio como un signo que está dotado de sentido y por lo tanto es portador de una estructura significante. El significado del silencio se ha estudiado desde diversas corrientes de la etnolingüística y la sociolingüística, pero en este trabajo es interesante el planteamiento que se hace respecto a la comunicación y a la función del silencio desde la escuela de Palo Alto a partir de mediados del S.XX. El pensamiento de Palo Alto liderado por pensadores como R. Birdwhistell, G. Bateson, E. Hall y E. Goffman, entre otros, intenta dar una respuesta a las premisas ofrecidas por parte del psicoanálisis y la psicología de la forma, que centran su interés en el inconsciente y en las percepciones. Por su parte estos autores norteamericanos prestan gran parte de su atención al contexto y a los patrones culturales donde se produce el hecho comunicativo. Desde esta perspectiva, la comunicación es pensada como un sistema que funciona más allá de la voluntad del interactuante, convirtiendo la ausencia de comunicación en una tarea imposible, ya que el sujeto constantemente está emitiendo mensajes a quienes le rodean. El actor social como miembro de un sistema cultural está inmerso en el proceso comunicativo del que forma parte activa. Y es por ello por lo que a pesar de que el silencio se manifieste mediante la ausencia de palabra en el acto comunicativo, no esta vacío de significado ni se puede separar del discurso. No es imperativo ni impulsor, sino que son los gestos corporales los que incitan a la interacción en el caso del intercambio sexual anónimo. Pero sin embargo, si que es un elemento clave que permite la interpretación de la acción llevada a cabo y facilita la comunicación corporal al permitir prestar una mayor atención al cuerpo.

Ángeles Marco (2001) advierte que el silencio no es un fenómeno que quede fuera de la comunicación, sino que por el contrario forma parte del proceso comunicativo de forma absoluta y necesaria. Es por ello por lo que está culturalmente pautado para cada contexto y cada sociedad, de la misma manera que lo están el resto de los actos comunicativos. Cualquier ejercicio de comunicación tiene necesariamente asociado un contexto cultural que lo enmarca para hacerlo legible e interpretable, de manera que tanto el lenguaje verbal como el no verbal responden a unas pautas establecidas socialmente. Por lo tanto, para analizar el silencio como fenómeno inscrito a un acto comunicativo deben de tenerse en cuenta todos los elementos que intervienen en él: emisor, receptor, canal y código. Evidentemente, el silencio, puede llevar a malentendidos, tergiversaciones o equivocaciones a causa de la ruptura en la relación emisor-receptor-canal-código, pero de la misma manera que sucede en la palabra, el lenguaje escrito o visual. Es por ello por lo que podemos afirmar que si entendemos la comunicación como una acción cultural, el silencio también forma parte de este proceso de comunicación social a pesar de su particularidad marcada por la ausencia de sonido.

Partimos pues de que el silencio tiene diferentes interpretaciones, reglas y significados en función del espacio en el que se produce. Es por ello por lo que en las zonas de cruising, adquiere un valor comunicativo propio con un significado 
compartido que queda sujeto a la interpretación de los participantes en la interacción sexual. En las zonas de intercambio sexual anónimo, tanto el emisor como el receptor interactúan desde la distancia mediante el uso del lenguaje no verbal y con unos códigos comunicativos que son marcadamente diferentes a los que utilizarían en otros escenarios de su vida social. A pesar de la ausencia de palabras, la comunicación entre los interactuantes fluye con relativa normalidad gracias a ese código compartido que permite interpretar las acciones y los mensajes emitidos por el resto de participantes con una finalidad claramente sexual. La comunicación en las zonas de cruising opera de manera similar a la que describe Goffman (1979) en las relaciones en público. Los implicados en el juego, ayudados por el lenguaje corporal informan a los otros desconocidos de sus intenciones, de manera que los receptores se ven obligados a responder a esta acción comunicativa y devolver al emisor una respuesta tanto en el caso de ser afirmativa como negativa.

En muchos otros espacios sociales el silencio podría ser causante de incomodidad para los presentes. Sin embargo en las zonas de cruising es posible una reunión de diez o más hombres en la que se sitúen los unos frente a los otros sin establecer ningún tipo de comunicación verbal durante más de quince minutos. Los participantes utilizan algunos movimientos corporales para generar información que les conduzca a la negociación sexual con el resto de los asistentes. "Hay un lenguaje no verbal que tiene que ser decodificado para poderlo entender. Yo me comunico mucho con el cuerpo y con la mirada"

El silencio resulta más cómodo para el intercambio sexual anónimo, se trata de una regla de conducta que no obliga a inventar historias ni a hacer esfuerzos para caer bien a las parejas sexuales, no hay que invitar a copas a nadie, ni perder demasiado tiempo en la interacción para saber si se obtendrá el resultado esperado. Sitúa a las personas en el tipo de lugar en el que están y ayuda a centrar la atención en el objeto del encuentro.

Yo lo entiendo, porque la gente hace sexo y quiere silencio. Sin hablar ni gritar. Ese no es ambiente para el sexo,... A mí me gusta el silencio. He tenido pensamientos muy profundos en estos lugares. Es como un tiempo para mi mismo. Donde puedo hacer algo sin discusiones.

Es sexo puro y duro. Porque el que espere encontrar por aquí el príncipe azul, pues no lo va a encontrar. Como no sea una casualidad, que a ver, dejaremos un $2 \%$ a la casualidad. Pero aquí la mayoría viene al aquí te pillo y aquí te mato. Y ¡adiós! Y si te has quedado contento y la otra parte también, si te ves otro día dices. Pues ¡vale!, ¡otra vez!.

El silencio permite una retirada fácil del territorio, las personas acostumbran a salir de la zona de cruising sin despedirse. Incluso un participante puede interrumpir el ritual de acercamiento, o el acto sexual en sí mismo, sin tener que dar ninguna justificación ni excusa a la pareja sexual. Autoriza a marcharse sin mediar palabra y retomar de nuevo la búsqueda en favor de otras parejas sexuales sin la necesidad de atender a la satisfacción de su pareja previa. De hecho, este tipo de 
interrupciones son muy comunes en la fase finalista de la interacción ritual, algunas personas acostumbran a abandonar el acto sexual después de haber pasado un tiempo con una misma pareja sexual y deciden reiniciar la búsqueda para poder tener un mayor número de contactos. Otras veces, algunos usuarios cuando han llegado a la eyaculación, abandonan al otro participante independientemente de la fase de excitación en que éste se encuentre. Es habitual que la persona que ya ha satisfecho los deseos sexuales, sin mediar palabra se suba los pantalones y deje a la pareja sexual del momento. He podido verificar como para resolver esta situación la persona abandonada, a veces, trata de encontrar a algún voyeur que estaba mirando su relación previa para intentar que éste pueda ayudarle a acabar la faena realizando una masturbación mutua, o enlazando con cualquier otra nueva relación sexual. En este sentido, gracias a la observación participante he podido comprobar como en algunas ocasiones, el participante que queda excitado tras un contacto sexual pero no ha llegado al orgasmo decide masturbarse hasta eyacular. O finalmente puede volver a la zona de encuentro con el objetivo de buscar otras posibles parejas sexuales con quienes terminar la acción sexual iniciada.

La noción de silencio tiene también un carácter prospectivo en las zonas de cruising, es decir, los usuarios deben guardar el secreto de aquella actividad una vez han salido de la zona de intercambio sexual, no hablar de ello, no vincularse a lo que allí sucede. Y de alguna manera, invisibilizarlo, contribuir a la visión de inexistencia, y por supuesto tampoco desvelar cualquier información que se ha podido obtener respecto a las personas que la frecuentan. Aunque cabe puntualizar que en algunas ocasiones diversos usuarios aseguran que han recibido amenazas por parte de los vecinos de la zona de cruising, e incluso cuentan historias de intentos de chantaje y extorsión. La práctica del cruising podemos decir que es también una práctica silenciosa en la medida en que no se puede hablar de ella para ocultar su existencia y a sus participantes.

A pesar de su eficacia para la consecución del doble objetivo de interacción sexual y anonimato, el silencio también puede generar problemas a la hora de pactar las negociaciones sexuales. Algunos participantes pueden continuar una dinámica sexual en la que no se sienten cómodos, pero prefieren atenerse a la situación por respeto a la norma social compartida con los otros jugadores que plantear los problemas que se generan en la interacción (Elwood, Greene, Carter, 2003). Este es el caso de la negociación en el uso del preservativo, por ejemplo. En silencio, este tipo de negociaciones resulta más complicada si la interacción se inicia sin la intención de mantener una relación con el uso del condón, de manera que los participantes para no cortar el ambiente sexual creado acceden a realizar prácticas sexuales sin protección.

Hombres que tienen sexo con hombres informaron que la norma del silencio en las saunas facilita la eficiencia y confidencialidad de los encuentros sexuales [...] esta norma comunicativa, sin embargo, en ocasiones limita la capacidad de los hombres que tienen sexo con hombres a negociar el uso del preservativo para el sexo anal, porque la negociación de un acto sexual específico, así como el uso del preservativo conlleva la violación inmediata de la norma subjetiva que requiere silencio en los encuentros sexuales. (Ellwood, Greene y Carter, 2003:290) 
La obligación de silencio también genera otros problemas relativos a las enfermedades de transmisión sexual, en la medida en que no se habla de ellas, se convierten en un hecho secundario, como si se tratase de algo inexistente, imposible, o inviable, lo que provoca que las personas dejen de atender y cuidar sus relaciones sexuales, ya que las preocupaciones por la salud tienen una importancia secundaria con respeto a la norma del silencio. En esta línea, Percy Fernandez-Dávila (2009) analiza como existen algunas condiciones sociales que hacen que hombres que tienen sexo con hombres decidan atender otras necesidades no-sexuales a pesar de que esto conlleve a implicarse en prácticas sexuales de alto riesgo.

La comunicación verbal, como hemos visto, no es un requisito indispensable para el encuentro de pareja sexual en las zonas de cruising. De hecho, el uso del lenguaje oral, fácilmente puede ser entendido como una intromisión en el espacio personal y visto como algo fuera de lugar.

La gente hace sexo, y quiere silencio. No hablar y gritar. Hablar no es un ambiente del sexo. Para eso tienes que ir al bar. Si estas al lado del árbol y tienes que hacer sexo, o algo un poco romántico. iQue no es tan romántico! (risas). Si hay mucha gente que hablan y grita pues el ambiente esta muy mal. Esto no es un club donde hay música muy fuerte.

Sin embargo, esto no significa que el lenguaje verbal esté alejada de manera determinante y definitiva del intercambio sexual anónimo. Como apuntan Douglas y Tewskbury (2008), cuando se recurre a su uso, normalmente es de forma secundaria y como complemento a los aspectos ya transmitidos a partir de la glosa corporal. En cualquier caso, no es habitual que se establezcan largas conversaciones entre los diferentes participantes y puntualmente algunos usuarios que se han encontrado anteriormente pueden iniciar una conversación que facilite la repetición de la relación sexual. No obstante, las señales verbales suelen trasmitir poca información respecto a los individuos, pero pueden facilitar el desarrollo del juego sexual. Normalmente este tipo de comunicación se establece a partir de frases cortas que se utilizan para tranquilizar o para excitar a la pareja sexual del tipo: “Vamos!”, “Está bien?”, "¿Cómo va?”, "¿Te gusta mi rabo?”, pero no se suelen utilizar para la iniciación del coqueteo.

En algunas ocasiones el lenguaje verbal también se utiliza para aclarar cuestiones que no han quedado resueltas a partir del lenguaje corporal, por ejemplo el rechazo de una pareja sexual. Normalmente a partir del lenguaje corporal las personas pueden rechazar la interacción sexual con otros participantes, pero en ocasiones algunos de ellos se vuelven insistentes y continúan con sus intentos para provocar el encuentro sexual. En este tipo de situaciones los participantes pueden recurrir al lenguaje verbal diciendo frases como: “iNo quiero nada!" o "iNo me apetece!”, quedando generalmente así resuelto el conflicto. En este sentido uno de los participantes entrevistados comentaba que "entrabas ahí, y si había cuatro viejos, los cuatro viejos no te dejaban en paz, persiguiéndote todo el rato, hasta el punto que les tenías que decir. - ¡Fuera! ¡Dejarme en paz! ¿no?-”. Otra de las reacciones para eludir un encuentro sexual se puede llevar a cabo marchando del espacio donde 
está siendo acosado, especialmente cuando la otra parte es excesivamente insistente incluso abordando un contacto físico rechazado.

Por otro lado, existen algunos participantes que acostumbran a establecer una comunicación verbal vinculada a la zona de cruising, pero fuera del área destinada al encuentro. Normalmente se da en las zonas menos concurridas, a la entrada de la zona destinada para la interacción. Los participantes suelen ser los mismos repetidamente e incluso se conocen de otros espacios de la vida social, como puede ser en el caso de las zonas rurales. De manera que el silencio en este tipo de lugares no cumple una función de anonimato, ya que a pesar del silencio, los usuarios conocen gran parte de las vidas personales del resto de participantes. En estos casos, las conversaciones acostumbran a ser de poca intensidad emocional (pero con gran carga sexual) y de tipo transitorio mientras se espera la llegada de nuevos usuarios. A pesar de este conocimiento previo, el silencio se mantiene a lo largo del proceso de negociación sexual tal y como ocurre en otros espacios de intercambio sexual anónimo, pero también se mantiene con respecto al resto de la esfera social. Es decir, a pesar de que unos participantes conozcan a los otros mantienen ese secreto compartido para evitar el descubrimiento de cada uno de ellos y así asegurar la demanda de anonimato.

Aunque el lenguaje verbal es escaso, existen otros recursos para comunicarse con el resto de los participantes. Algunos usuarios acostumbran a hacer ruidos tales como chasquidos o silbidos con la boca para provocar que le presten atención otros usuarios. Se trata de sonidos breves y diferenciados en el tiempo en función del fluido de personas que merodean por el parque. Este tipo de sonidos también son utilizados en varias ocasiones por aquellos que están un poco más retirados de la zona de intercambio, y no se les puede ver a primera vista. Igualmente, un cigarrillo encendido o el mechero también indica en la oscuridad los lugares donde se encuentran otros participantes. Además, y a pesar de que el ejercicio sexual normalmente se lleva a cabo en silencio, existen usuarios que emiten algunos gemidos cuando están llevando a cabo el acto sexual. Estos gemidos tienen un carácter muy tímido y solamente alcanza a escucharlos las personas que están llevando a cabo el acto sexual próximo, fundamentalmente tienen por objeto mantener la excitación personal y de la pareja sexual.

Sin lugar a dudas, la capacidad de los individuos para saber comunicar con su cuerpo los intereses sexuales es imprescindible para maximizar el beneficio en los encuentros anónimos. Así, gracias a las diferentes acciones que los usuarios emprenden, se comunica la disponibilidad para el juego sexual convirtiendo cada movimiento en un elemento importante para la obtención del beneficio final. A partir del cuerpo, los jugadores informan de sus intenciones y también interpretan las de cada uno de los participantes presentes. Por lo tanto, el ámbito de lo visual es fundamental para la interacción. Los diferentes movimientos corporales se resignifican para dotarles de un contenido comunicativo que los participantes utilizan con esta doble finalidad que por un lado es sexual pero también anónima. Tal y como señala Goffman (1982 [1971]) respecto al dialecto corporal, podemos verificar que los participantes de las zonas de cruising interactúan entorno a un discurso 
convencionalizado y normativo en el que los usuarios están obligados a transmitir determinadas informaciones a partir de la glosa corporal para poder ser integrados en la actividad del cruising. En este sentido, los actores del intercambio sexual anónimo deben transmitir los datos más relevantes a través de su cuerpo que les puedan comprometer con la finalidad del encuentro. De manera, que en cada espacio y fase de la comunicación existen unas reglas que distribuyen y regulan el proceso comunicativo del acuerdo sexual.

Este acto comunicativo se establece a partir de algunas normas de sincronización que permiten obtener la atención del receptor del mensaje. Birwhistell (1982[1970]) defiende la existencia de una sincronía interaccional mediante la cual los cuerpos que interactúan en una acción comunicativa oscilan a un mismo ritmo, de manera que las partes participan de un sistema de interacción que esta por encima de su comportamiento individual. Es por ello por lo que los comportamientos comunicativos perceptibles por la vista presentan unas propiedades analógicas diferentes a las que se podrían observar en una comunicación centrada en las percepciones del oído. En las zonas de cruising, la ausencia de palabra permite esta sincronía entre los cuerpos y una comunicación cargada de significado que conduce al acuerdo sexual. La sincronía se establece gracias al soporte de la mirada mantenida entre los usuarios, la muestra de los genitales al receptor del mensaje y el acercamiento pausado que permiten pautar los acuerdos de la relación para finalmente llegar a la acción sexual. Así, los participantes que se mueven por el parque no tienen por objeto desplazarse de un lugar a otro, sino que por el contrario realizan ciertos actos corporales para que puedan ser percibidos por los otros como mensajes para la seducción sexual. Los usuarios acostumbran a caminar sosegadamente, con paradas constantes transmitiendo una actitud merodeadora, curiosa y atenta a todo aquello que sucede a su alrededor, donde pueden mirar sin permiso, o mejor dicho donde el permiso para mirar a los otros esta otorgado sin cuestionamiento. Los participantes quieren ver y ser vistos para poder iniciar a la interacción sexual y mediante la sincronización corporal adquieren la atención de otros participantes y pueden promocionar la oportunidad para el gozo sexual. Además, a través del modo de caminar también se puede transmitir la urgencia de la interacción, ya que algunas personas por ejemplo, caminan rápidamente buscando interacciones constantes con la finalidad de agilizar la llegada al acto sexual, mientras que otras dedican largo tiempo a la búsqueda de la pareja sexual más adecuada. Es decir, algunos de los participantes de los que caminan rápido por la zona de cruising buscan la interacción con una buena parte de los hombres que están allí, sin realizar un ejercicio de selección, inician el acercamiento con muchos de los hombres independientemente de la edad, el origen o el aspecto físico. Tratan de estimular a cualquiera que acepte la interacción para llegar así al acto sexual lo antes posible. Mientras que otros participantes, se mantienen pacientes y solo responden a las interacciones sexuales cuando se encuentran ante un sujeto que les resulta atractivo o deseable.

Una gran parte de los movimientos se inician conscientemente con el objetivo de provocar una respuesta por parte del resto de participantes, pero otros no siempre pueden llevarse a cabo de manera consciente y decidida como es el caso del modo de caminar o de apoyarse en los árboles. Cada uno de los usuarios, poco a 
poco, adiestra su cuerpo en este modo de interacción con la finalidad de adaptarse al medio y a los patrones comunicativos compartidos para obtener el beneficio sexual. Cabe puntualizar que en algunos lugares de cruising de las grandes ciudades, los usuarios pueden llegar a ser de muy diferentes contextos culturales, lo que provoca que en algunas ocasiones la adaptación y adiestramiento del cuerpo resulte más complicada en el caso de aquellos que tampoco forman parte de la "cultura madre" de la zona de intercambio. Es decir, los participantes que con un recorrido cultural más afín al territorio donde se lleva a cabo la interacción, también tienen más facilidad para incorporar las técnicas de adiestramiento de las zonas de cruising en ese lugar determinado. Sin embargo es necesario añadir que la garantía de éxito no solo corresponde a una estrategia comunicativa capaz de adaptarse al nuevo medio a partir de una lectura corporal, sino que también al valor asignado en función de otros criterios como son étnicos o de edad que segrega a aquellos cuerpos deseables de los que no lo son. De manera que las personas de orígenes culturales diferentes a los de la zona de cruising tienen mayores dificultades para acceder al sexo por dos motivos fundamentales. Por un lado a causa de las diferentes maneras de entablar la comunicación no verbal con los otros participantes. Y por el otro, porque son objeto de rechazo a causa de su diferencia étnica. "Yo hace muchos años que ya no voy [refiriéndose al parque de Montjuïc]. Porque lo que había pues eran también mucho marroquí, y a ver, yo no soy racista. Pero no me fío".

Los participantes aprenden las técnicas corporales, para hablar en los mismos términos que Marcel Mauss (1991[1950]), que les permiten convertirse en individuos sociales gracias a este aprendizaje corporal. Cuando el individuo ha comprendido y asume la preconfiguración corporal necesaria para este tipo de interacciones, también puede predecir e interpretar los movimientos de los demás y rentabilizar la interacción.

El individuo debe transmitir un claro mensaje corporal para que pueda ser interpretado por los otros, este mensaje debe ser favorable y no llevar a la confusión. Los signos corporales que provocan la interacción suelen ser más extremos que en otros escenarios sociales, y los participantes suelen utilizar los genitales como elemento emisor del mensaje que muestra el interés en la interacción. Así, la polla toma un valor simbólico de gran importancia convirtiéndose en un elemento de comunicación supremo que permite mostrar y reconocer los intereses del participante que recurre a ella en el proceso comunicativo. El valor polla podría pensarse de un modo similar al planteamiento sobre el falo que se hace desde el psicoanálisis en la medida en que se convierte en un instrumento productor de significados. La polla es el valor que identifica la masculinidad de los presentes y que a su vez evita cualquier posible duda entorno a la identidad de género de los participantes. Así mismo, el culo también tiene un lugar simbólico en el intercambio sexual anónimo y es percibido con frecuencia como un espacio instrumental para el placer, que a la vez es degradado como orificio penetrante del que los participantes no acostumbran a mostrar orgullo, sino que por el contrario desvela una especie de fenimización de la que gran parte de los hombres quiere rehuir, porque como apunta Bourdieu "la peor humillación para un hombre es verse convertido en mujer" (2005 [1998]:36). 
El valor polla en las zonas de cruising contribuye a una masculinidad maximizada que se representa a partir de los juegos corporales de los participantes. En algunas ocasiones las relaciones sexuales entre hombres son vividas con miedo de ser asociadas con "lo femenino" (Guasch, 1995; Eribon, 2001[1999], Coll-Planas, 2010; Lores, 2012). La homosexualidad masculina se ha asociado constantemente al ámbito de lo femenino, lo que fácilmente se puede entender como una rebaja en la escala del poder estructurado desde la sociedad patriarcal. Ser homosexual, de alguna manera, es no ser hombre del todo, ya que "el certificado de masculinidad" pasa por la reivindicación de la heterosexualidad. Las mujeres tienen un valor simbólico inferior al de los hombres dentro de la esfera pública y privada. Es por ello por lo que entendemos que estos excesos de masculinidad que podemos observar en las zonas de cruising a partir de la relevancia que toma la polla, son ejercicios simbólicos que tienen por objeto no perder su estatus de superioridad a pesar de que se esté llevando a cabo una práctica "denigrante" para algunos participantes. Es decir, podemos pensar que se trata de demostrar a los demás, pero también a uno mismo, que a pesar de que se esté llevando a cabo una práctica que no está legitimada ni incluida en el manual del "buen macho", continua siendo un hombre "de verdad" y es por ello por lo que debe mantener los privilegios asignados como hombre a pesar de su "desviación". En este sentido, el valor polla, adquiere ese significado dominante que se otorga al órgano genital; el tamaño, la anchura y la forma anatómica permite señalar a los "más hombres" de la zona de cruising. La polla así, se presenta como un órgano incontrolado, inconsciente e irreprimible que de manera autónoma responde a un deseo sexual sin domesticar. Siguiendo a Sabuco i Cantó y Valcuente del Rio (2003: 145-146), podríamos apuntar que la representación del hombre es la de un ser pollado,en la que el órgano genital se convierte en un elemento constituyente de persona. Donde el pene es el requisito previo para tener poder. Así, los hombres pueden controlar su cuerpo, pero no los mandatos de su polla.

Los canales de acercamiento a los otros cuerpos desconocidos son mucho más permisivos que en otros lugares de interacción social. Es habitual, por ejemplo, que cuando una persona se acerca a otra, sin ningún tipo de miramiento ni vergüenza le toque la polla, o incluso le haga un breve masaje en el órgano genital y continúe con su trayecto sin más detención. Si embargo, en el supuesto de que este tipo de contactos físicos se pudiese producir en otros escenarios públicos entre desconocidos, posiblemente generaría la ira del otro ciudadano mientras que en las zonas de cruising son una costumbre. En la interacción, el cuerpo toma una forma distinta a la de otros espacios públicos. Se piensa como una especie de instrumento de intercambio de placeres, donde la intimidad no esta reservada a ciertas partes corporales, como ocurre en otros lugares, sino que se reduce a la identidad de las personas. Es decir, cuanto menos reveladora de datos personales sea la interacción sexual, más íntima se considera. El cuerpo es pues un objeto de intercambio, de más o menos libre disposición al resto de participantes. Posiblemente un viandante que se presenta fortuitamente en una zona de intercambio sexual anónimo sin ser conocedor de este lenguaje corporal extremo, pronto será descubierto por el resto de participantes que con toda seguridad se mantendrán al margen de su presencia e intentarán evitar el 
contacto con el intruso. Pero también, del mismo modo, la persona que no conoce el lenguaje corporal de la zona de cruising, fácilmente puede quedar sobresaltado al hallarse en este espacio donde es posible que no entienda lo que sucede, pero sin duda, el modo de comportamiento de las personas que se encuentran a su alrededor puede resultarle francamente sospechoso.

\section{Conclusiones}

El silencio se presenta como una herramienta capaz de construir diferentes significados en función del contexto, el sujeto o grupo social que recurre a él para significarlo. En el caso del sexo anónimo, el silencio es un utensilio indispensable para la comunicación que conduce al acto sexual. Se trata de una estrategia que permite garantizar el anonimato, agilizar la interacción y orientarla hacia el sexo. Pero este silencio, llamémosle estratégico, esta condicionado por dos elementos que deben ser tomados en cuenta. Por un lado, se trata de un silencio que parte del ocultamiento de los sujetos que participan en este tipo de actividades. Y por el otro lleva a la asunción del mandato social que obliga a la invisibilización de todas aquellas realidades ajenas a las lógicas socio-sexuales imperantes.

De esta manera, estrategia, ocultamiento e invisibilización son tres elementos que se deben tener en consideración a la hora de interpretar el silencio en el marco del sexo anónimo. No solo por la forma en la que se manifiestan, sino también en la que se interrelacionan, ya que aunque se puedan visualizar de modo separado, están fuertemente vinculados. Pero sobre todo, permiten constatar la supervivencia y mantenimiento de las lógicas sexuales hegemónicas a pesar de la apertura de ciertos escenarios de visibilidad homosexual de las últimas décadas. El silencio vinculado a la práctica del sexo anónimo da cuenta de una realidad social ambivalente en la que se ha producido una amplificación de ciertas visibilidades homosexuales, las cuales se han construido como nuevas hegemonías. Pero sin embargo se mantiene en un lugar "silencioso" aquellas otras que no han podido adquirir el valor de la aceptación social. De esta manera, se produce una ruptura en aquello que se ha llamado comunidad homosexual, ya que no todas las prácticas sexuales, ni todos los cuerpos, ni todos las formas de deseo han encontrado un lugar de representación, respetabilidad y aceptación por el conjunto de la sociedad.

\section{Referencias bibliográficas}

ALBUQUERQUE DE BRAZ, Camilo

2003 "Vestido de Antropólogo: nudez e corpo em clubes de sexo para homens". Bagoas. Estudos gays, gêneros e sexualidades. 2:3, 75-96

BAILEY, Robert

1999 Gay politics, Urban polities: Identity and Ecónomics in the Urban setting. New York: Columbia University Press.

BÉRUBÉ, Allan

1996 "The history of gay Bathhouse", en D. Bedfellows (Ed.), Politicing public sex. Boston: South End Press, 187-220. 
BIRWHISTELL, Ray

1982 [1970] "Un ejercicio de kinésica y de lingüistica: la escena del cigarrillo" en G. Bateson, R. Birwhistell, E. Goffman [et al.] (Eds.) La nueva comunicación. Barcelona: Kairos, 166-197.

BOSWELL, John

1992 [1980] Cristianismo, tolerancia social y homosexualidad: los gays en Europa occidental desde el comienzo del cristianismo hasta el siglo XIV. Barcelona: Muchnik.

BOURDIEU, Pierre

2005 [1998] La dominación masculina. Barcelona: Anagrama.

COLL-PLANAS, Gerard; MISSÉ, Miquel

2009 Diagonóstico de las realidades de la población LGTB de Barcelona. Instituto de Gobierno y Políticas Públicas: Ayuntamiento de Barcelona.

COLL-PLANAS, Gerard

2010 La voluntad y el deseo. La construcción social del género y la sexualidad: el caso de lesbianas, gays y trans. Barcelona: Egales.

DELPH, Edward W.

1978 The silent community. Public homosexuality encounters. California: Sage publications.

DOUGLAS, Brian; TEWKSBURY, Richard

2008 "Theaters and sex: an examination of anonymous sex encounters in an erotic oasis". Deviant behavior, 29:1, 1-17.

DUGGAN, Lisa

2002 "“The new homonormativity: the sexual politics of neoliberalism" en R. Castranovo, D. Nelson (Eds.) Materializing Democracy: Toward a Revitalized cultural Politics. Durham y Londres: Duke University Press. 175-194.

ELWOOD, William N.; GREENE, Kathryn; CARTER, Karen K.

2003 "Getlemen don't speak: communication norms and condom use in bathhouses". Journal of Applied Communication Research, 31 (4): 277-297.

ERIBON, Didier

2001[1999] Reflexiones sobre la cuestión gay. Barcelona: Anagrama.

2004 Una moral de lo minoritario. Variaciones sobre un tema de Jean Genet. Barcelona: Anagrama.

FERNANDEZ-DÁVILA, Percy

2009 "Necesidades no-sexuales que motivan a un grupo de hombres que tienen sexo con hombres a involucrarse en prácticas sexuales de alto riesgo ". Forum: qualitative social research, 10 (2): art.21.

FOUCAULT, Michael

2005[1976] Historia de la sexualidad. La voluntad de saber. Madrid: Siglo XXI. 
FLOWERS, Paul; HART, Graham; MARRIOTT, Claire

1999 "Constructing sexual health. Gay men and "risk" in the context of a public sex environment". Journal of health Psychology, 4 (4): 483-495.

GOFFMAN, Erving

1979 Relaciones en público: microestudios del orden público. Madrid. Alianza.

1982 [1981] “Compromiso" en G. Bateson, R. Birwhistell, E. Goffman [et al.] (Eds.) La nueva comunicación. Barcelona: Kairos, 287-298.

GUASCH, Oscar

1991 La sociedad Rosa. Barcelona: Anagrama.

2002 "Prologo", en Herdt, G. y Koff, B. Gestión familiar de la homosexualidad, Barcelona: Bellaterra, 13-17.

HAHN, Pierre

1979 Nos ancêtres les pervers. La vie des homosexuels sous le second empire. Paris: SEPC.

HERDT, Gilbert; KOFF, Bruce

2002 Gestión familiar de la homosexualidad, Barcelona: Bellaterra.

HIGGINS, Ross

1999 "Bath, bushes, and belonging: Public sex and gay community in pre-Stonewall Montreal”. en W. Leap (Ed.), Public sex / Gay space. Nueva York: Columbia University Press. 187-202.

HUBER, Jonathan D. y KLEINPLATZ, Peggy

2002 "Sexual Orientation Identification of men who have sex with men in public settings in Canada", Journal of Homosexuality, 42 (3): 1-20.

HUMPHREYS, Laud

1975 [1970] Tearoom trade. Impersonal sex in public places. New york: Enlarged.

LORES, Fernando

2012 Deseo y placer: anotaciones anotaciones antropológicas a una teoría de la contaminación y de los cuidados sexuales. Tesis doctoral. Facultad de Ciencias Políticas y Sociología. Universidad Complutense de Madrid.

MARCO FURRASOLA, Ángeles

2001 Una antropología del silencio. Un estudio sobre le silencio en la actividad humana. Barcelona: PPU.

MAUSS, Marcel

1991 [1950] Sociología y antropología. Madrid: Tecnos.

MIRA, Alberto

2007[2004] De Sodoma a Chueca: Historia cultural de la homosexualidad en España 1914-1990. Madrid: Egales. 
PICHARDO GALÀN, José Ignacio

2009a Entender la diversidad familiar. Relaciones homosexuales y nuevos modelos familiares. Barcelona: Bellaterra.

2009b "(Homo)sexualidad y familia: cambios y continuidades al inicio del tercer milenio". Política y sociedad, 2009, 46 (1-2): 143-160.

PLATERO MÉNDEZ, Raquel

2008 Herramientas para combatir el bullying homofóbico. Madrid. Talasa.

PONTE, Meredith R.

1974 "Life in a parking lot: An ethnography of a Homosexual Drive-in" en J. Jacobs (Ed.) Deviance: Field studies and self-Disclosures. California: National Press Book, 7-29.

REECE, Michael y DODGE, Brian

2004 "Exploring the physical mental and social well-being of gay and bisexual men who cruise for sex on a college campus". Journal of homosexuality, 46 (1): 111-136.

RUBIN, Gayle

1989[1984] "Reflexiones sobre el sexo: notas para una teoría radical de la sexualidad" en C. Vance, (comp.) Placer y peligro. Explorando la sexualidad femenina. Madrid: Talasa.

SABUCO I CANTÓ, Assumpta; VALCUENDE DEL RIO, José Maria

2003 "La homosexualidad como representación hiperbólica de la masculinidad”, en J.M. Valcuende del Rio, y J. Blanco López (Eds.), Hombres. La construcción cultural de las masculinidades. Madrid: Talasa.

SEDGWICK, Eve K.

(1998 [1990]) Epistemologia del armario. Barcelona: Ediciones de la Tempestad.

SILABIS, Michael

1999 "Paris" en D. Higg (Ed.), Queer sites. Gay urban histories since 1600. Londres: Routledge.

TATTELMAN, Ira

1999 "Speaking to the Gay bathhouse: communicating in sexually charged spaces" en W. Leap (Ed.), Public sex / Gay space. Nueva York: Columbia University Press. 71-94.

TEWKSBURY, Richard

2008 "Finding erotic oasis:locating the sites of men's sam-sex anonymous sexual encounters". Journal of Homosexuality, 55 (1): 1-19.

TROIDEN, Richard R.

1974 "Homosexual encounters in a Higway rest stop", en E. Goode y R.R. Troiden (eds.), Sexual Deviance and Sexual deviant. Nueva York: William Morrow and company, 211-228.

VÁZQUEZ GARCÍA, Francisco; CLEMINSON, Richard

2010[1997] Los invisibles. Una historia de homosexualidad masculina en España 1850-1939. Granada: Comares. 
VÁZQUEZ GARCÍA, Francisco; MORENO MELGÍBAR, Andrés

1997 Sexo y razón. Una genealogía de la moral sexual en España. Madrid: Akal.

VILLAAMIL PEREZ, Fernando; JOCILES RUBIO, Maria Isabel

2008 "Diferencias y desigualdades entre los HSH usuarios de locales comerciales de encuentro sexual: algunas contribuciones a las estrategias comunitarias de prevención del VIH". Etnográfica. 12 (2): 185-321.

2011 "Risk and community: The impact of HIV among gay in Madrid. The case of sex clubs". Sexualities, 14 (5): 580-596.

WESTON, Kath

2003 [1997] Las familias que elegimos. Lesbianas, gays y parentesco. Barcelona: Bellaterra.

WITTIG, Monique

2006[1992] El pensamiento heterosexual y otros ensayos. Madrid: Egales

WEEKS, Jeffrey

1993 El malestar de la sexualidad. Madrid: Talasa.

WEINBERG, Martin S.; WILLIAMS, Colin J.

1975 "Gay Baths and the social organization of impersonal sex". Social Problems. 23 (2): 124-136. 